divided patients into two groups, first one diagnosed prior to the pandemic (before march 2020), and the second - diagnosed during pandemic-associated restrictions period. Both groups were compared according to FIGO (International Federation of Gynaecology and Obstetrics) staging and presence of symptoms (hydrothorax and ascites). Statistical analysis was performed with logistic regression analysis. Statistical significance level was set at 0,05 .

Result(s)* Before the pandemic, 47 patients were admitted with a median age of 61 . During the pandemic, there were 30 newly diagnosed patients with a median age of 59. In both groups the most common type of cancer was high grade serous adenocarcinoma $(61,7 \%$ and 60,0\%, respectively). Patients with an advanced OC (FIGO stage III and IV) accounted for $57,4 \%$ in the pre-pandemic group, while in the second group patients with advanced cancer accounted for $66,7 \%$. Although the percentage was higher in the second group, the logistic regression analysis did not confirm the impact of pandemic on more frequent occurrence of FIGO III $(p=0,17)$ and IV $(p=0,81)$ diagnosis. Ascites was found in $29,8 \%$ of patients before and $30 \%$ during pandemic. Hydrothorax was observed in 14,9\% of patients in the first group and $26,7 \%$ in the second one. Logistic regression analysis revealed no influence of pandemic on percentage of symptomatic patients $(p=0,91$ for ascites and $p=0,18$ for hydrothorax).

Conclusion* The number of newly diagnosed OC patients was lower during the pandemic than in the preceding year. Without regard to healthcare availability, OC remains the disease which is diagnosed in the advanced stage.

\section{WHY GYNAE-ONCOLOGY? DETERMINING THE PREFERENCES OF IRISH TRAINEES IN BECOMING A GYNAE-ONCOLOGIST}

K Mulligan*, S Murphy, C Thompson. The Mater Misericordiae University Hospital, Ireland

\subsection{6/ijgc-2021-ESG0.330}

Introduction/Background* There are currently 156 Obstetric $\&$ Gynaecology (O\&G) trainees in Ireland. Fourteen (8\%) of whom have expressed an interest in undertaking subspecialist training to pursue a career in Gynaecological Oncology (GO). The training pathway in Ireland is currently eight years before there is an opportunity to subspecialise and there are less than 10 Gynaecological Oncology Specialist Registrar (SpR) rotational positions per year on the national training scheme. This provides a challenge for Irish trainees who wish to gain exposure to Gynaecological Oncology from an early stage in their career.

Methodology We wished to assess the current opinions/preferences of trainees who had commenced or wished to commence subspecialist training in GO. A SurveyMonkey was distributed to this cohort and responses received anonymously. Particular focus was placed on the trainees preferred structure of fellowship training and subsequently on what they felt would be their preferred structure of Consultant job specification once qualified.

Result(s)* Of the 14 respondents, $15 \%$ were training at GO fellowship level, 45\% were at SpR level and 40\% were Basic Specialist Trainees $(<3$ years training in O\&G). There were a number of reasons for trainees being interested in pursuing a career in GO. 100\% became interested due to the surgical procedures involved, $60 \%$ found the disease conditions interesting and $42 \%$ of trainees felt GO was 'the best way of receiving high volume surgical training in O\&G'.

Regarding research, all trainees wish to undertake formal research with $40 \%$ wishing to complete an $\mathrm{MD}$ and $30 \%$ a $\mathrm{PhD}$. Formally recognised training was felt essential with $50 \%$ feeling that ESGO was their preferred accreditation. Regarding duration, $50 \%$ felt a two year fellowship should be sufficient for gaining clinical experience. Only $25 \%$ of trainees wished to train less than full time.

Need for more surgical training workshops and increased dedicated Gynaecological training time at $\mathrm{SpR}$ level in order to obtain experience at an earlier stage in their career trajectory was highlighted.

Conclusion* This small cohort study highlights the challenges faced by Irish trainees who wish to become Gynaecological Oncologists. Nationally there is already progress on increasing simulation training, development of mentoring and more accredited training positions.

\section{Ovarian cancer}

\section{ANALYSIS OF PATIENTS WHO DERIVED EXCEPTIONAL BENEFIT FROM RUCAPARIB MAINTENANCE TREATMENT FOR HIGH-GRADE OVARIAN CANCER IN THE PHASE 3 ARIEL3 STUDY}

\begin{abstract}
${ }^{1} \mathrm{~J}$ Ledermann*, ${ }^{2} \mathrm{~A}$ Oza, ${ }^{3} \mathrm{D}$ Lorusso, ${ }^{4} \mathrm{C}$ Aghajanian, ${ }^{5} \mathrm{~A}$ Oaknin, ${ }^{6} \mathrm{~A}$ Dean, ${ }^{7} \mathrm{~N}$ Colombo, ${ }^{8} \mathrm{~J}$ Weberpals, ${ }^{9} \mathrm{~T}$ Kwan, ${ }^{10} \mathrm{R}$ Coleman. ${ }^{1}$ UCL Cancer Institute, University College London and UCL Hospitals, Department of Oncology, London, UK; ${ }^{2}$ Princess Margaret Cancer Centre, University Health Network, Division of Medical Oncology and Hematology, Toronto, Canada; ${ }^{3}$ Multicenter Italian Trials in Ovarian Cancer and Gynecologic Malignancies and Gynecologic Oncology Unit, Fondazione IRCCS, Istituto Nazionale dei Tumori, Milan, Italy*; ${ }^{4}$ Memorial Sloan Kettering Cancer Center, Department of Medicine, New York, USA; ${ }^{5}$ Vall d'Hebron Institute of Oncology (VHIO), Hospital Universitari Vall d'Hebron, Vall d'Hebron Barcelona Hospital Campus, Gynecologic Cancer Program, Barcelona, Spain; ${ }^{6}$ St John of God Subiaco Hospital, Department of Oncology, Subiaco, Australia; 'University of Milan-Bicocca and European Institute of Oncology (IEO) IRCCS, Gynecologic Cancer Program, Milan, Italy; ${ }^{8}$ Ottawa Hospital Research Institute, Division of Gynecologic Oncology, Ottawa, Canada; ${ }^{9}$ Clovis Oncology, Inc., Molecular Diagnostics and Translational Medicine, Boulder, USA; ${ }^{10}$ Department of Gynecologic Oncology and Reproductive Medicine, University of Texas MD Anderson Cancer Center, Houston, TX, USAt; *Affiliation where the work was conducted; current affiliation: Gynecologic Oncology Unit, Fondazione Policlinico Universitario A. Gemelli IRCCS and Scientific Directorate, Rome, Italy; †Affiliation where the work was conducted; current affiliation: US Oncology Research, The Woodlands, TX, USA
\end{abstract}

\subsection{6/ijgc-2021-ESG0.331}

Introduction/Background* ARIEL3 is a placebo-controlled randomized trial of the PARP inhibitor rucaparib as maintenance treatment in high-grade ovarian cancer (HGOC) patients who responded to the latest line of platinum therapy (NCT01968213). Rucaparib improved progression-free survival (PFS) across all predefined subgroups. Here, we present an exploratory analysis of characteristics associated with exceptional benefit from rucaparib.

Methodology Between 7 April 2014, and 19 July 2016, 564 patients were randomized 2:1 to rucaparib $600 \mathrm{mg}$ BID or placebo. As of 31 December 2019 (data cutoff), 33/375 (9\%) and $1 / 189(0.5 \%)$ patients were still ongoing and receiving rucaparib or placebo. Molecular features (genomic alterations 


\begin{tabular}{|c|c|c|c|}
\hline \multirow[b]{2}{*}{ Baseline characteristics } & \multicolumn{3}{|c|}{ Rucaparib } \\
\hline & $\begin{array}{c}\text { Exceptional benefit } \\
n=79\end{array}$ & $\begin{array}{l}\text { Short-term subgroup } \\
n=64\end{array}$ & $\begin{array}{l}\text { Odds ratio } \\
(95 \% \mathrm{Cl})\end{array}$ \\
\hline No measurable disease & $58(73 \%)$ & $26(41 \%)$ & $4.0(2.0-8.0)^{a}$ \\
\hline Complete response to latest platinum & $31(39 \%)$ & $13(20 \%)$ & $2.5(1.2-5.3)^{a}$ \\
\hline Penultimate platinum-free interval $>12 \mathrm{mo}$ & $55(70 \%)$ & $30(47 \%)$ & $2.6(1.3-5.2)^{\mathrm{a}}$ \\
\hline BRCA mutation & $46(58 \%)$ & $12(19 \%)$ & $6.0(2.8-13.3)^{\mathrm{a}}$ \\
\hline BRCAwt/LOH-high & $18(23 \%)$ & $19(30 \%)$ & $0.7(0.3-1.5)$ \\
\hline BRCAwt/LOH-low & $8(10 \%)$ & $28(44 \%)$ & $0.14(0.1-0.4)^{\mathrm{a}}$ \\
\hline BRCAwt + RAD51C/D mutation ${ }^{\mathrm{b}}$ & $6(8 \%)$ & 0 & NA \\
\hline BRCAwt + high BRCA1 promoter methylation ${ }^{c}$ & $6 / 25(24 \%)$ & $7 / 47(15 \%)$ & $1.8(0.5-6.0)$ \\
\hline
\end{tabular}

and BRCA1 promoter methylation) and baseline clinical characteristics were compared between patients who derived exceptional benefit (PFS $\geq 2$ years), and those with disease progression on first scan ( $\approx 12$ weeks; the short-term subgroup) within each treatment arm.

Result(s)* Of 564 patients, a greater percentage of rucaparib vs placebo patients showed exceptional benefit: 79/375 (21\%) in the rucaparib arm and 4/189 (2\%) in the placebo arm (including 26/375 [7\%] patients in the rucaparib arm and 1/ 189 [0.5\%] patient in the placebo arm with PFS $>4$ years as of the data cutoff date). Within the rucaparib arm, exceptional benefit patients had more favourable clinical prognostic factors at baseline versus the short-term subgroup (table 1). Although BRCA (BRCA1 or BRCA2) mutations were enriched in the rucaparib exceptional benefit subgroup, 33/79 (42\%) of these patients were BRCA wild type. Patterns of enrichment varied among other biomarkers. Overall trends were similar in the placebo arm.

Conclusion* Exceptional benefit in ARIEL3 was more common in, but not exclusive to, patients with favourable clinical characteristics and known mechanisms of sensitivity to a PARP inhibitor. Our results suggest rucaparib can deliver exceptional benefit to a diverse set of patients with HGOC.

\section{ABSTRACT WITHDRAWN}

\section{ROLE OF ULTRASOUND IN STAGING OF OVARIAN CANCER}

A Elagwany. Alexandria Faculty of Medicine, Egypt

\subsection{6/ijgc-2021-ESG0.332}

Introduction/Background* Ovarian cancer is usually staged surgically . Radiologic staging using CT scan along ca125 is used before surgery. Ultrasound can be used in staging before surgery related to the advances in technology and machines. Ultrasonography has some advantages over CT and MRI being a dynamic procedure during which the assessment of sliding and vascular patterns are evaluated. Vascularity can assess the nature of the lesion and the borders. We are trying here to spot the lights over this staging along with a pictorial illustration of different stages.

Methodology With advances in machines, sonography has become an accurate staging method for early and advanced gynecological tumors. Other techniques, such as computed tomography and magnetic resonance imaging, can be used as an adjunct to ultrasound in specific cases. There are still limitations in some cases In such cases, a complementary imaging method or diagnostic laparoscopy can be used. Staging of ovarian cancer is as follows. Stage 1 with ovarian involvement, stage 2 with pelvic affection, stage 3 with abdominal and nodal affection and stage 4 with extra abdominopelvic metastasis.

Result(s)* . Ultrasound is main imaging method for the differential diagnosis of benign and malignant ovarian tumors based on IOTA classification .Ultrasound can be used to detect early and locally advanced cancers along with detection of extrapelvic tumor in the abdominal cavity in the form of parietal or visceral carcinomatosis, omental and/or mesenteric infiltration. we can proceed with pattern recognition by expert, deal as malignant or apply IOTA adnex model. Secondly, we proceed with Staging of ovarian cancers .

Conclusion* Ultrasound can be used in staging ovarian cancer iota malignant cases and after pattern recognition with some limitation.

\section{DOSE MODIFICATION FOR CHINESE PATIENTS ON NIRAPARIB MAINTENANCE TREATMENT FOR PLATINUM-SENSITIVE RECURRENT OVARIAN CANCER: A POST HOC ANALYSIS}

${ }^{1} \mathrm{Z} \mathrm{Liu}^{*},{ }^{2} \mathrm{X}$ Wu, ${ }^{3} \mathrm{~J} \mathrm{Zhu},{ }^{4} \mathrm{R}$ Yin, ${ }^{5} \mathrm{~J}$ Yang, ${ }^{6} \mathrm{~J} \mathrm{Liu,}{ }^{7} \mathrm{~J} \mathrm{Wang},{ }^{8} \mathrm{~L} \mathrm{Wu},{ }^{9} \mathrm{~T}$ Tan, ${ }^{9} \mathrm{~J}$ Dong. ${ }^{7}$ The First Hospital of Jilin University, Department of Oncology, Changchun, China; ${ }^{2}$ Fudan University Shanghai Cancer Center, Department of Gynecologic Oncology, Shanghai, China; ${ }^{3} \mathrm{Cancer}$ Hospital of the University of Chinese Academy of Sciences (Zhejiang Cancer Hospital), Department of Gynecologic Oncology, Hangzhou, China; ${ }^{4}$ West China Second University Hospital, Sichuan University, 1.Department of Obstetrics and Gynecology 2. Key Laboratory of Birth Defects and Related Diseases of Women and Children (Sichuan University), Ministry of Education, Chengdu, China; ${ }^{5}$ Peking Union Medical College Hospital, Chinese Academy of Medical Sciences and Peking Union Medical College, Department of Gynecologic Oncology, Beijing, China; ${ }^{6}$ Sun Yat-sen University Cancer Center, Department of Gynecologic Oncology, Guangzhou, China; ${ }^{7}$ Hunan Cancer Hospital, the Affiliated Cancer Hospital of Xiangya School of Medicine, Central South University, Department of Gynecologic Oncology, Changsha, China; ${ }^{8}$ National Cancer Center/Cancer Hospital, Chinese Academy of Medical Sciences and Peking Union Medical College, Department of Gynecologic Oncology, Beijing, China; ' Zai Lab (Shanghai) Co., Ltd, RandD Department, Shanghai, China

\subsection{6/ijgc-2021-ESGO.333}

Introduction/Background* In the NOVA trial of niraparib maintenance treatment (MT) in platinum-sensitive recurrent ovarian cancer (PSROC), dose reduction due to treatmentemergent adverse events (TEAEs) occurred in $66.5 \%$ of patients initiating niraparib at $300 \mathrm{mg}$. Based on NOVA RADAR analysis, subsequent individualised starting dose (ISD) 